\title{
A new friction condition identification approach for wheel-rail interface
}

\author{
Altan Onat ${ }^{\mathrm{a}}$, Petr Voltr ${ }^{\mathrm{b}}$ and Michael Lata ${ }^{\mathrm{a}}$ \\ a Department of Transport Means and Diagnostics, Jan Perner Transport Faculty, University \\ of Pardubice, Czech Republic; ${ }^{b}$ Educational and Research Centre in Transport, Jan Perner \\ Transport Faculty, University of Pardubice, Czech Republic
}

\begin{abstract}
In recent years, there has been an increasing interest in designing intelligent vehicles such that they can take necessary actions according to the environmental changes around them and they can inform decision makers about these changes. For safer and cheaper transport, dynamic modelling of these vehicles and identification of such changes in environment based on these models plays an important role. In this study, a sigma point Kalman filter based scheme (i.e. joint unscented Kalman filter) is proposed to estimate maximum friction coefficient as a parameter in wheel-rail interface. This estimation scheme uses interpretation of lateral and yaw dynamic response of a wheelset to identify maximum friction coefficient. This joint unscented Kalman filter based approach provides information about the friction conditions in wheel-rail interface without post-processing of estimated data.
\end{abstract}

\section{KEYWORDS}

joint unscented Kalman filter; friction identification; parameter estimation; railway vehicles; wheelset dynamics; wheel-rail contact.

\section{Introduction}

The dynamic response of a railway vehicle depends upon the interaction of wheel and rail. When the friction condition in this interface changes, it directly affects dynamic response of the vehicle. This response is a key to have faster and safer vehicles.

Friction condition must be considered during the traction or braking phase such that if it is not taken into consideration, phenomena like wear, instability and unwanted delays in schedule may occur. Furthermore, important track signals can be passed due to low friction levels which can cause accidents. Therefore, there is a need of having information about the friction conditions in wheel-rail interface.

The use of model based filtering for railway vehicles is not a new concept. Nevertheless, the focus of such model based filtering schemes is mostly estimating the conditions of primary and secondary suspension systems. This is called condition monitoring and this scheme helps to arrange more efficient maintenance schedules [1-3].

Previously reported studies reveal that different friction (i.e. adhesion) conditions cause different dynamic responses of wheelset. First attempts for detection of low adhesion is presented by Charles and Goodall [4] and Charles et al. [5], and it is stated that estimating creep coefficients from dynamic response is problematic, and for accurate estimation, more knowledge about the wheel-rail contact must be included. 
Charles et al. [5] conclude that instead of having information of exact friction coefficient, an indication of low adhesion conditions is sufficient and this can be achieved by estimating contact forces and processing them. Xia et al. $[6,7]$ present an inverse model based contact forces estimation scheme that can be used for adhesion condition identification purpose. A multiple model estimation scheme for adhesion condition estimation is demonstrated by Hussain et al. [8] and instead of estimating contact forces or moments, the residuals of a set of Kalman filters are analysed. These filters are operating in different points of creep force - creepage curves. This proposed multiple model scheme also requires a post-processing stage to interpret adhesion conditions from residuals of Kalman filters. Besides, the wheelset model [8] has coned wheels without lateral primary suspension, which is not realistic, as across the globe worn wheel profiles are used recently. A model based adhesion estimation scheme, which is based on the analysis of contact forces and moments, is reported by Ward et al. [9] . The dependence of creep forces and moments upon the level of track irregularity is emphasized as it makes difficult to interpret adhesion conditions without prior knowledge of track irregularity. However, without prior knowledge of the track irregularity level, adhesion conditions can be estimated by using a post-processing method based on the eigenvalue analysis [9]. A non-model based method is proposed by Hubbard et al. [10] which is based on the comparison of the dynamical responses of leading and trailing wheelsets. In this non-model based estimation scheme, the yaw positions of the leading and trailing bogies are used. A search to find a cross correlation of all dynamic variables of leading and trailing bogies is conducted and it is concluded that biggest change in correlation is in the yaw angle and it can be used for interpretation [10]. Spiryagin et al. [11] introduce a method to identify adhesion condition which is based on the relationship between adhesion coefficient and slip for different friction conditions. This method uses the vertical and tractive force estimators for a locomotive to determine traction coefficient and then estimates the friction conditions by using the slip obtained from a slip estimator [11].

The main problem in model based estimation schemes is to decide a model which represents the physical system exactly in each condition. Dynamic modelling of railway vehicles for estimation purposes is not an exception. Especially, a model of a contact is vital to understand the interaction between wheel and rail. As stated in a recent survey of wheel-rail contact models by Meymand et al. [12], the most accurate solutions are proposed by Kalker $[13,14]$ for normal and tangential contact problem, but these solutions are computationally expensive to use in vehicle dynamics simulations. Most important requirement for a contact model is that it should be accurate and as computationally simple as possible. For the solution of the normal contact problem, theory of Hertz [15] is still used in up-to-date commercial multi-body simulation (i.e. MBS) tools and theory of Hertz is the simplest and fastest solution for the normal contact problem. On the other hand, other theories for normal contact problem mentioned by Meymand et al. [12] are more accurate than the theory of Hertz, whereas they require more computational power. In order to find contact forces, Kalker [16] also presents a method, namely Fastsim, which is the slightly modified version of his simplified theory. Thus, it can also be used for practical applications. Bosso et al. $[17,18]$ demonstrate such a real time contact module which leads to the possibility of using complex simulation models in diagnostic or control systems. This contact module and most of the up-to-date commercial multi-body system tools consider Fastsim as a solution method for the tangential problem. Although there are significant improvements for contact mechanics [12], even implementation of these simplified algorithms can be computationally expensive. Zhou et al. [19] use a medium-sized field-programmable 
gate array (i.e. FPGA) based multiprocessor system to overcome this disadvantage. In this study, theory of Hertz [15] is considered for the solution of normal contact problem and the analytical method proposed by Polach [20,21] is used for the solution of tangential problem. This analytical method for the solution of tangential problem is 3 to 8 times faster than Fastsim [20].

Especially at very low adhesion conditions, because of the decreasing creep forces, the restorative force due to the gravitational stiffness term becomes dominant force with respect to creep forces at wheel-rail contact [22]. Therefore, modelling errors due to linear contact approximation cause problems in order to identify very low adhesion conditions. Based on a nonlinear contact approximation, a comparison study between lateral dynamic responses of two degree of freedom dynamic model of a wheelset and MBS model is provided by Hubbard et al. [22]. It is concluded and shown that a two degree of freedom dynamic model of a wheelset is sufficient to capture lateral dynamic response of a wheelset with respect to lateral track irregularities.

To the authors' knowledge, the present work is the first one step estimation scheme proposed in related literature by using a joint unscented Kalman filter for estimation of maximum friction coefficient from dynamic response of a wheelset. The filtering method used in this work, namely unscented Kalman filter (i.e. UKF), dates back to the study by Julier et al. [23] and based on the seminal work of Kalman [24]. Kalman filter is optimal in sense of expectations (i.e. mean) and it uses the fact that conditional distributions of Gaussian random process are Gaussian. Kalman filter can only be applied to linear (or linearized) systems successfully. Another type of Kalman filter, namely extended Kalman filter (i.e. EKF), can be used for estimation of nonlinear systems and it is based on system linearization. EKF requires Jacobian matrix for the nonlinear system. Besides, even in linearizable systems, calculation of Jacobian matrix is difficult and error prone operation. In order to overcome these mentioned drawbacks, related with linearization and Jacobian matrix, unscented transformation (i.e. UT) is proposed. A very first explanation of this transform is given by Julier et al. [23]. In UKF, states and parameters are again represented by Gaussian random variables from a set of carefully chosen sample points [25]. These sample points are named as sigma points. Various versions and details of sigma point Kalman filters are reported by Van Der Merwe [26]. UT is based on the fact that it is easier to approximate probability distributions than approximating an arbitrary nonlinear function [27]. Chosen sigma points are subjected to a nonlinear transformation (UT) via nonlinear function of the system in order to obtain transformed points. By using the statistical properties of the transformed points, mean and covariance can be calculated for estimation. UKF seems as a similar approach to particle filters [26], but it exhibits several differences. Firstly, rather than particles, sigma points are chosen deterministically according to given mean and covariance. As a result, higher order information can be extracted by considering small amount of points. The important points of UKF are given by Julier et al. [27] as: firstly, algorithm includes finite number of points so that algorithm can be used as black box filtering to calculate estimated quantities when a model is given with defined inputs and outputs. This is the property in this study, which leads to the estimation of the quantity (e.g. maximum friction coefficient) in wheel-rail interface. The second important point is that the computational complexity of UKF is similar to that of EKF. Thirdly, UKF can be used with discontinuous transformations. Readers are referred to the studies by Kandepu et al. [28] and Matzuka et al. [29] which explain the application of UKF in nonlinear dynamic systems for state and parameter estimation. Additionally, a review of performance measures for such kind of filters are provided by Haug [30]. 


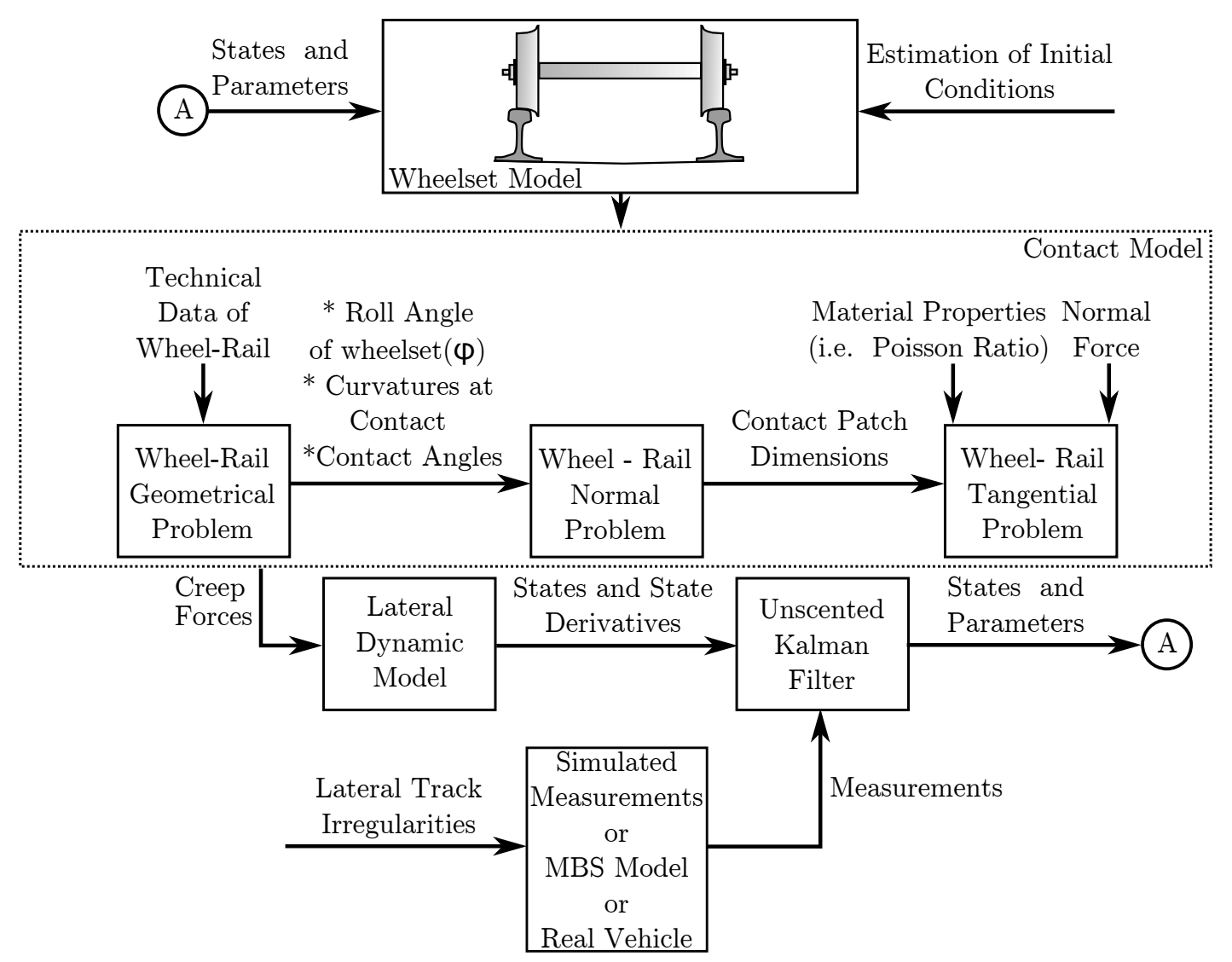

Figure 1.: General structure of estimation

This estimation approach can be used to obtain a friction map of different rail sections or to alert driver. The general structure of this study can be seen in Figure 1. This article is organized as follows: In Section 2, the contact model, which is used to solve wheel-rail geometrical, normal and tangential problem, is described shortly. In Section 3, the dynamic model, considered in this study, is explained. In Section 4, the dynamic response of the wheelset is given with respect to a defined input and track irregularities are defined. Section 5 covers brief information about UKF, results and their discussion.

\section{Contact model}

The wheel, considered in this study, has a new S1002 profile and rail has a UIC60E1 profile with 1:40 inclination. This wheel-rail pair is widely used across the Europe. Technical details and related data can be found about this wheel-rail profile in related European standards. Detailed explanations of the contact model are presented by Onat et al. [31]. Therefore, a brief description of this contact model is given here.

In order to solve geometrical problem, lateral position of wheels and rails are used, and then curvatures and conicity angles of wheel-rail are obtained, accordingly. As wheelset shifts laterally, especially in the vicinity of flange contact, considerable roll angle occurs which changes the contact point compared to the case when no roll angle 
is assumed. The semi-analytical method to find roll angle of the wheelset reported by $\mathrm{Li}[32]$ is used in this work. This semi-analytical method considers the equality of vertical distances at contact points on the right and left wheel. Let $d_{\min _{r}}$ and $d_{\text {min }_{1}}$ be the minima of vertical distances between wheels and rails on the right and left side, respectively. If the respective minima occur in the contact points, then these two minima should be equal. If they are not equal, roll angle of the wheelset must be adjusted. Suppose $d_{m_{i n}}>d_{m i n_{l}}$, then the wheelset must be rotated clockwise by an angle

$$
\Delta \theta=\frac{d_{\text {min }_{r}}-d_{\min _{l}}}{y_{\min _{l}}-y_{\min _{r}}}
$$

where $y_{\text {min }_{r}}$ and $y_{\text {min }_{l}}$ are the lateral positions of the minima. Rotation must be repeated until these two minima are equal in terms of the tolerance $\epsilon_{t o l}$. In this study, the value of the tolerance is taken as $10^{-4}$. Therefore, the roll angle can be calculated as a sum of the incremental rotations

$$
\theta=\theta_{0}+\sum_{i=1}^{k} \Delta \theta_{i}
$$

where $k$ is the number of rotations and $\theta_{0}$ is the initial roll angle. In this study, initial roll angle is accepted zero for all lateral shifts of the wheelset.

Quasi-elastic contact search method, reported by Arnold and Netter [33] and Schupp et al. [34], is used for contact locus search. Elastic contact search method is more realistic approach, but it requires the use of finite element methods. Therefore, the computational complexity is high. Quasi-elastic contact search method uses the weighted average of the distance function in the area of contact patch. The maximum deformation occurs at the contact patch and for other distant points, deformation decreases. The assumption here is that the relationship between deformations of both surfaces is exponential. An illustration of the parameters used in quasi-elastic contact search can be found in Figure 2. The weight function with respect to the distance function is given as

$$
w\left(s, z_{\text {wheel }}, z_{\text {rail }}\right)=\exp \left(\frac{-d\left(s, z_{\text {wheel }}, z_{\text {rail }}\right)}{\epsilon}\right)
$$

where $\epsilon$ is the regularization parameter. Regularization parameter is chosen so that vertical displacement of the wheel has the same size as the elastic deformation in pure elastic normal contact model as stated by Arnold and Netter [33]. In this study, it is chosen as $2 \times 10^{-5}$ which is within the range of appropriate values emphasized by Arnold and Netter [33]. Furthermore, $s$ given in Equation 3, is defined as $s=y-y_{c}$, where $y_{c}$ is the $y$ coordinate of the rigid contact point. The new contact location $\bar{s}$ in terms of $s$ is defined as

$$
\bar{s}=\frac{\int_{s_{\min }}^{s_{\max }} s \cdot w\left(s, z_{w h e e l}, z_{\text {rail }}\right) d s}{\int_{s_{\min }}^{s_{\max }} w\left(s, z_{\text {wheel }}, z_{\text {rail }}\right) d s} .
$$




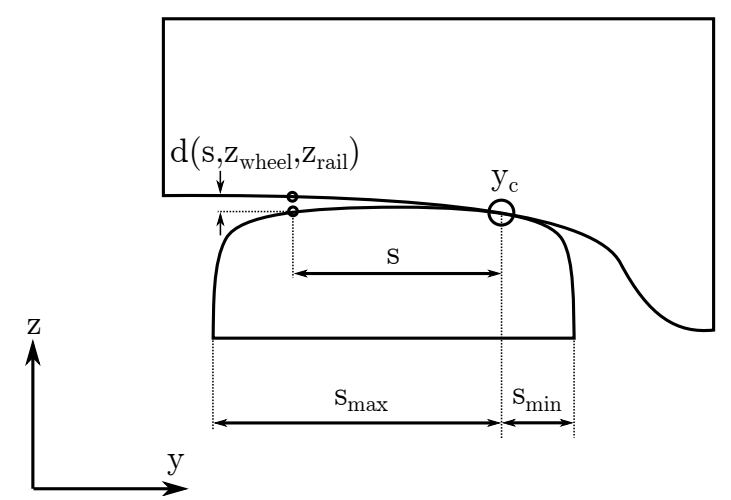

Figure 2.: Parameters used in quasi-elastic contact search

In order to solve wheel-rail normal problem, theory of Hertz [15] is used. For fast calculation, an approximation function is reported by Shabana et al. [35] to obtain tabulated coefficients given by Hertz [15]. In this work, the numerical solution of elliptical integrals are used to find contact patch by using theory of Hertz. Details of this methodology to find contact patch are presented by Onat et al. [31]. Dependent on how many terms are used in the solution of elliptical integrals, result can be more accurate while having close computational complexity to the approximation functions. In order to validate results of the normal problem and contact search, results provided by Gensys [36] are considered. Results can be seen for the contact patch semi-axes ratio in Figure 3. Biggest difference between results in Figure 3 is seen in the area of switching from wheel tread to the flange and in the flange contact. Additionally, it is a well - known fact that theory of Hertz does not provide accurate results in case of flange contact where contact angle changes substantially within the same contact patch as stated by Burgelman et al. [37]. The non-conformal contact assumption of Hertz fails in case of flange contact and flange contact occurs especially in the arcs of turnouts and in curves. For such cases, different contact models must be used [37]. In this study, since the level of track irregularities does not cause a flange contact and straight track is assumed, it is concluded that theory of Hertz is sufficient. Although it is not stated by Gensys [36], a different method might be used in case of flange contact similar to method presented by Burgelman et al. [37].

A creep force model is required to solve tangential problem. An experimentally validated creep force model is used in this work [21]. Besides, spin is an important phenomenon in the solution of wheel-rail contact problem. In this study, the effect of spin on lateral creep force is also considered and details of how spin causes an increase in lateral tangential force are expressed by Polach [20]. In order to calculate creep forces, it is a well - known fact that tabulated coefficients [13], namely Kalker coefficients, are required. These coefficients are tabulated with respect to the ratio of contact patch semi-axes and combined Poisson's ratio of wheel-rail. A polynomial fit for this table is reported by Iwnicki [38]. However, polynomial fit [38] assumes that Poisson's ratio of steel is close to 0.27 and polynomial equations are given only with respect to contact patch semi-axes ratio. In this work, Poisson's ratio of the wheel-rail is considered as 0.28 and the polynomial fit proposed by second author is used. This polynomial fit consists also Poisson's ratio as a variable. Polynomial fit for the Kalker coefficient in the longitudinal direction, which is considered in this study, is presented by Onat et al. [39]. Additionally, coefficients in other directions have similar polynomial structure. Minimum ratio of semi-axes in these polynomial 


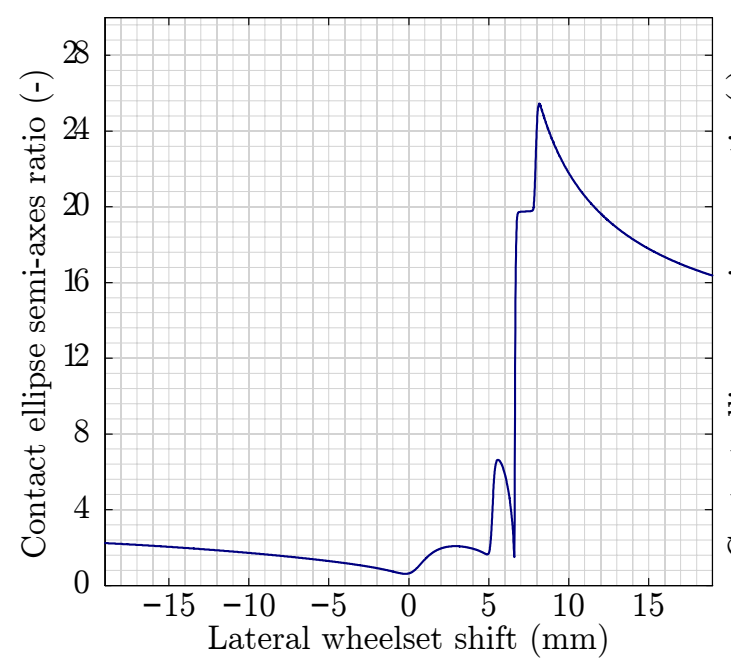

(a)

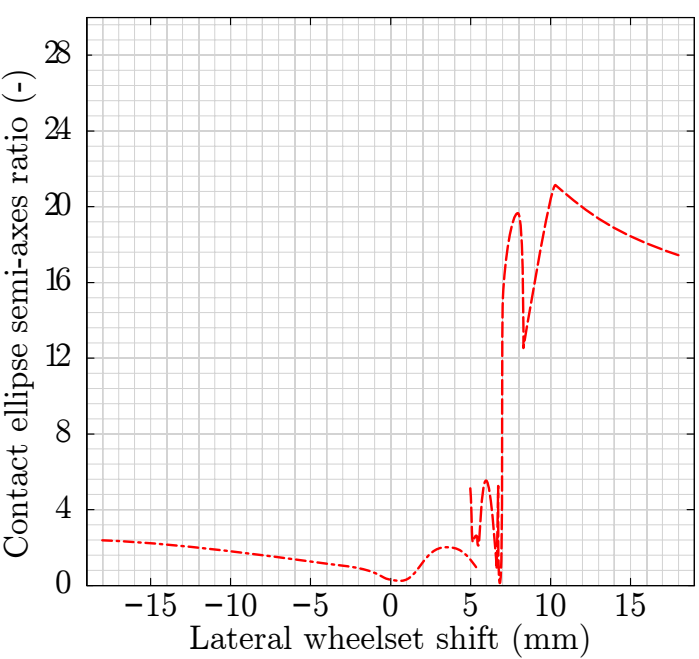

(b)

Figure 3.: Comparison of (a) result of this study (b) result of multi-body simulation tool Gensys, adapted from [36]

Table 1.: Creep force model parameters

\begin{tabular}{clcccc}
\hline Parameters & Definitions & Dry & Wet & Low & Very low \\
\hline$\mu_{0}$ & $\begin{array}{l}\text { Maximum friction coefficient } \\
\text { at zero slip velocity }\end{array}$ & 0.5 & 0.2 & 0.08 & 0.04 \\
$\mathrm{~A}$ & $\begin{array}{l}\text { Ratio of friction coefficients } \\
\mu_{\infty} / \mu_{0}\end{array}$ & 0.4 & 0.4 & 0.4 & 0.4 \\
$\mathrm{~B}$ & $\begin{array}{l}\text { Coefficient of exponential fric- } \\
\text { tion decrease }(\mathrm{s} / \mathrm{m})\end{array}$ & 0.6 & 0.2 & 0.2 & 0.1 \\
$k_{A}$ & $\begin{array}{l}\text { Reduction factor in the area } \\
\text { of adhesion }\end{array}$ & 1 & 1 & 0.6 & 0.3 \\
$k_{S}$ & $\begin{array}{l}\text { Reduction factor in the area } \\
\text { of slip }\end{array}$ & 0.4 & 0.4 & 0.2 & 0.1 \\
\hline
\end{tabular}

fits is 0.04 and maximum ratio is 25 . After obtaining Kalker coefficients, by using the creep force model with the assumption of decreasing friction coefficient with increasing slip proposed by Polach [21], tangential forces in longitudinal and lateral direction is calculated. The parameters for the creep force and friction model are selected similar to given by Charles et al. [5] and can be found in Table 1. In Table 1, it should be noted that decreasing friction causes a decrease in initial gradient of creep force-creep curve. Therefore, in order to be consistent with the real situation, different reduction factors $k_{A}$ and $k_{s}$ are selected with decreasing friction condition.

It is a known fact that dry or wet friction conditions do not constitute problem for braking, whereas low and very low friction conditions do. Especially in the design of braking systems across Europe, coefficient of friction of 0.15 is assumed (generally with some exceptions) such that the required braking performance must be achieved for any value of coefficient friction which is greater or equal to 0.15 , as stated in the article 4.2.4.6.1 of European standard [40]. 


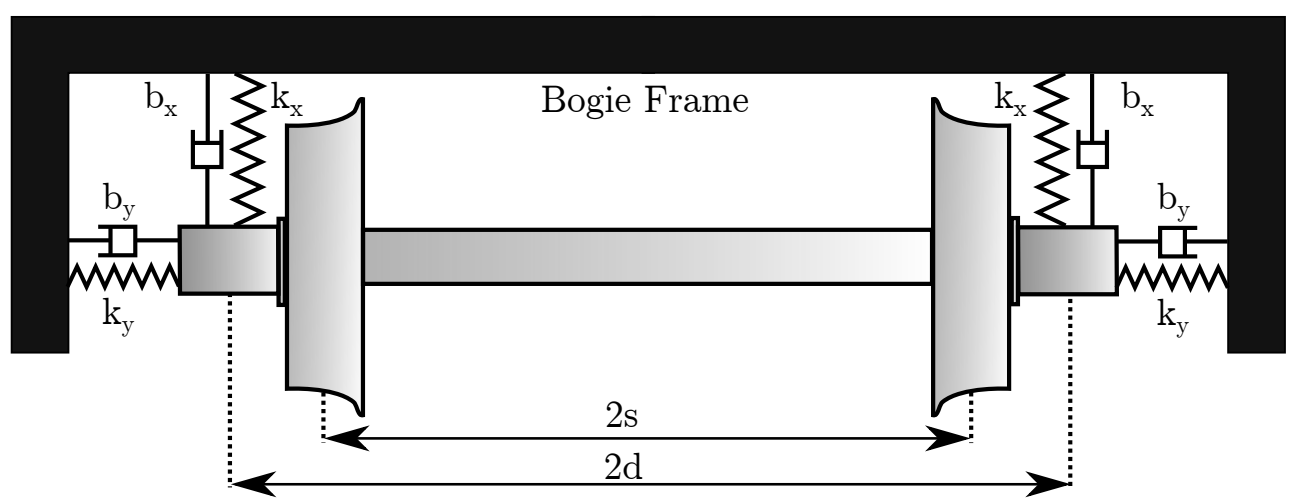

(a)

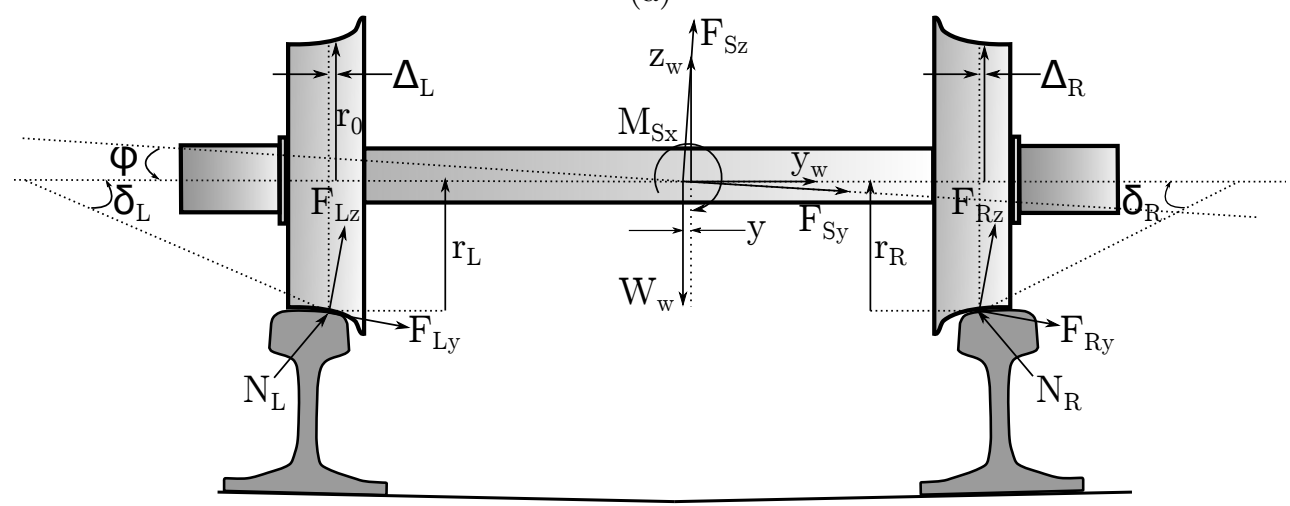

(b)

Figure 4.: Plan view model of a wheelset (a) top view and (b) front view

\section{Dynamic model of wheelset}

The dynamic model of wheelset reported by Garg and Dukkipati [41], namely planview dynamic model, is used in this study. An illustration of wheelset can be found in Figure 4, which shows the interconnections between wheelset and bogie, and forces acting on the wheelset. It has been shown by Hubbard et al. $[10,22]$ that this model is sufficient to track lateral dynamic response of wheelset in comparison with simulations by using Vampire multi-body simulation package.

Differential equations for the lateral dynamic model of the wheelset can be given as follows:

$$
\begin{aligned}
m_{w} \ddot{y} & =F_{L y}+F_{R y}+N_{L y}+N_{R y}+F_{S y}, \\
I_{w x} \ddot{\psi} & =-I_{w y} \frac{V}{r_{0}} \dot{\phi} \dot{y}+\left(R_{L x} F_{L y}-R_{L y} F_{L x}\right)+\left(R_{R x} F_{R y}-R_{R y} F_{R x}\right) \\
& +R_{L x} N_{L} \sin \left(\delta_{L}-\phi\right)-R_{R x} N_{R} \sin \left(\delta_{R}-\phi\right)+M_{L z}+M_{R z}+M_{S z}
\end{aligned}
$$

In Equation $5 \mathrm{~b}, R_{L x}, R_{R x}, R_{L y}, R_{R y}$ are the components of position defined in inertial fixed frame (i.e. equilibrium axis). Definitions of these vectors and other terms in these equations are provided by Garg and Dukkipati [41]. Additionally, in order to allow wheelset to follow track irregularities, instead of a rigid support between wheelset and superstructure, this model includes a laterally constrained suspended mass $\left(m_{m}\right)$ 
which can be given by

$$
m_{m} \ddot{y}_{m}=-F_{S y} .
$$

In this equation, $F_{S y}$ is the acting lateral force due to the lateral suspension elements, and $M_{S z}$ in Equation 5b is the acting yaw moment due to the longitudinal suspension elements, which are defined by

$$
\begin{aligned}
F_{S y} & =-2 k_{y}\left(y-y_{m}\right)-2 b_{y}\left(\dot{y}-\dot{y}_{m}\right), \\
M_{S z} & =-2 k_{x} d^{2} \psi-2 b_{x} d^{2} \dot{\psi},
\end{aligned}
$$

where $d$ is the half of the distance between suspension centres and can be seen clearly in Figure 4. These forces and moments are defined in the axis of wheelset centre, and they must be transformed into the fixed inertial frame by using transformation matrices presented by Garg and Dukkipati [41]. Besides, in order to calculate creep force components, definitions of creepages are required. The lateral and longitudinal creepages defined by Onat et al. [39] are used in this study.

\section{Dynamic response of a wheelset due to track irregularities}

In this section, the lateral and yaw dynamic response of the wheelset are shown with respect to a $5 \mathrm{~mm}$ step input under different maximum friction coefficient conditions. Hereby, step input represents the instantaneous change in lateral alignment of track with a magnitude of $5 \mathrm{~mm}$. Parameters, which are used for simulations in this study, can be found in Table 2 .

Table 2.: Wheelset dynamic model parameters

\begin{tabular}{clc}
\hline Parameters & Definitions & Values \\
\hline$V$ & Longitudinal Velocity of Wheelset & $40 \mathrm{~m} / \mathrm{s}$ \\
$r_{0}$ & Nominal Radius of Wheels & $0.46 \mathrm{~m}$ \\
$s$ & Half of the Tape Line Distance & $0.75 \mathrm{~m}$ \\
$G$ & Shear Modulus of Rigidity of Wheel and Rail & $8 \times 10^{10} \mathrm{~Pa}$ \\
$Q$ & Wheel Load & $55 \mathrm{kN}$ \\
$k_{y}$ & Lateral Spring Stiffness & $2 \times 10^{6} \mathrm{~N} / \mathrm{m}$ \\
$k_{x}$ & Longitudinal Spring Stiffness & $4 \times 10^{6} \mathrm{~N} / \mathrm{m}$ \\
$b_{y}$ & Lateral Damping Coefficient & $1 \times 10^{3} \mathrm{Ns} / \mathrm{m}$ \\
$b_{x}$ & Longitudinal Damping Coefficient & $1 \times 10^{3} \mathrm{Ns} / \mathrm{m}$ \\
$d$ & Half of the Distance Between Suspension Centers & $1 \mathrm{~m}$ \\
$m_{w}$ & Mass of the Wheelset & $1813 \mathrm{~kg}$ \\
$m_{m}$ & Suspended Mass & $6241 \mathrm{~kg}$ \\
$I_{w y}$ & Moment of Inertia of Wheelset Around y Axis & $112 \mathrm{kgm}^{2}$ \\
$I_{w z}$ & Moment of Inertia of Wheelset Around x Axis & $1120 \mathrm{kgm}^{2}$ \\
\hline
\end{tabular}

Step input is applied after 1 second, and it can be easily seen in Figure 5 that the wheelset has a unique response with respect to the different maximum friction conditions with same lateral alignment irregularity. This is the main reason to conclude 
that maximum friction coefficient can be estimated at one step by using a model based filtering method (i.e. UKF). Similar responses of identical dynamic model of a wheelset with respect to different maximum friction conditions are reported by Hubbard et al. $[22]$ and also a decrease in yaw angle with respect to decreasing friction coefficient is observable [22]. It is indicated that in order to estimate extremely low levels of adhesion by processing estimated contact forces and moments, a linear contact approximation is insufficient [22]. Therefore, a non-linear contact model is also considered in this study.

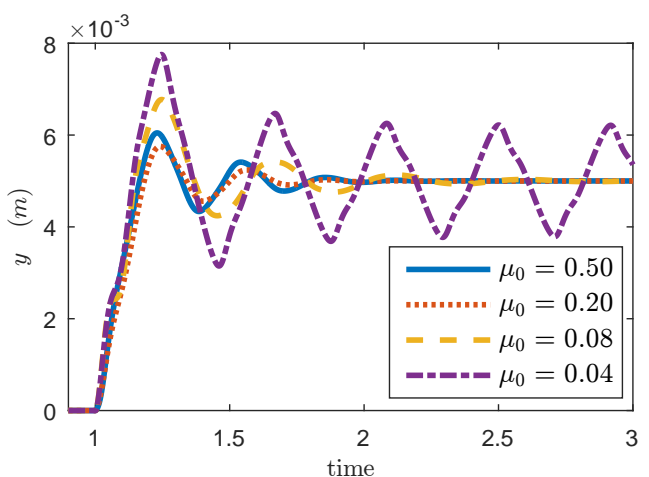

(a)

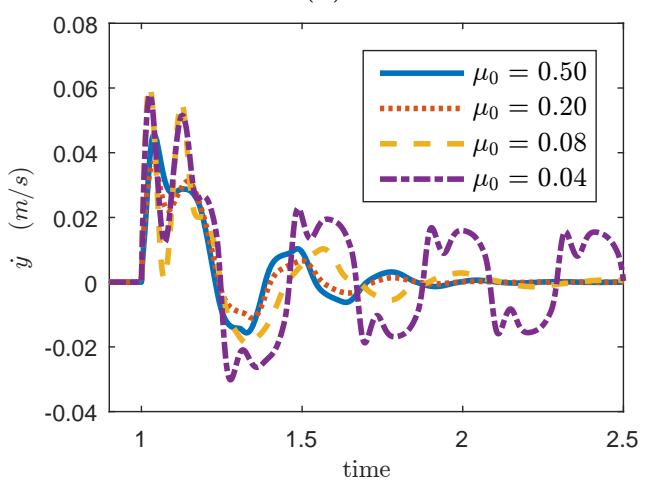

(c)

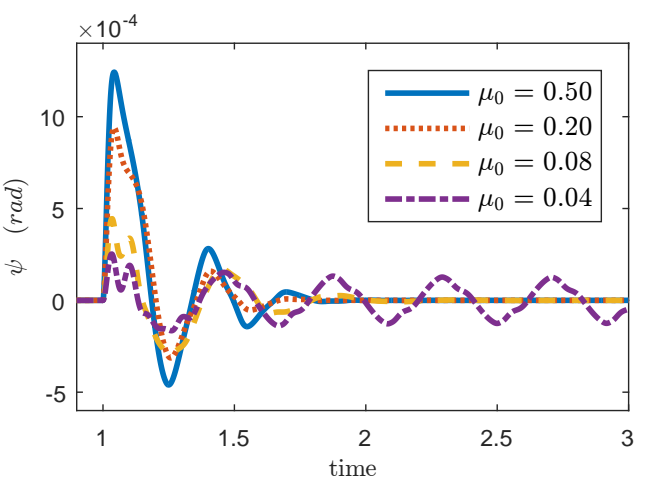

(b)

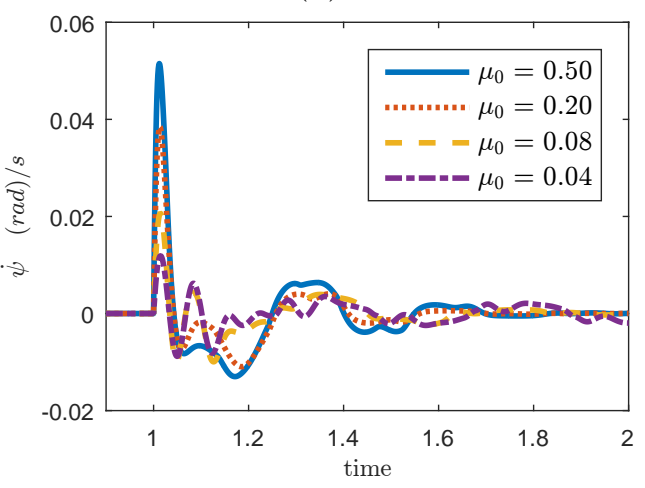

(d)

Figure 5.: Dynamic response of the wheelset with respect to different friction conditions: (a) lateral Shift, (b) yaw angle, (c) lateral velocity and (d) yaw velocity

In order to be consistent with the real situation, instead of randomly generated step inputs for lateral alignment irregularity, measurements from a track section (3 $\mathrm{km}$ length) between Choceň-Dobřikov, Czech Republic is considered in this study. The details of how track irregularities are measured with respect to related standards given by Karis [42]. In this study, since only lateral and yaw dynamics are considered, the lateral alignment irregularities are used. The statistical characteristics of the considered irregularity are presented in Table 3.

Table 3.: Lateral track irregularity statistical characteristics

\begin{tabular}{cccc}
\hline Maximum irregularity $(\mathrm{mm})$ & Minimum irregularity $(\mathrm{mm})$ & Standard deviation $(\mathrm{mm})$ & Variance $\left(\mathrm{mm}^{2}\right)$ \\
\hline $3.8 \mathrm{~mm}$ & $-3.3541 \mathrm{~mm}$ & $0.8103 \mathrm{~mm}$ & $0.9002 \mathrm{~mm}^{2}$
\end{tabular}

Similar to the study by Pombo et al. [43], track irregularities are also parametrized 
here by using shape preserving cubic splines with respect to the track length. In Chapter 14 of the study reported by Iwnicki [38], lateral alignment irregularity can be derived from the lateral shift of the rails as

$$
y_{i r r}=\frac{y_{L}+y_{R}}{2}
$$

An illustration of lateral alignment irregularity is presented by Iwnicki [38].

The dynamic model used in this study is validated by means of comparison with respect to a model of multi-body simulation software VAMPIRE, in Section 3.2 of the study given by Hubbard et al. [22]. Both dynamic models considered in this study and in [22] are similar, instead, as well as a nonlinear contact patch, model presented here includes a nonlinear creep force model. Additionally, a comparison of a linear plan view suspension model and the 3D suspension model of VAMPIRE is provided by Hubbard et al. [10] and concluded in model development and validation section of same study that linear plan view suspension forces sufficiently match the suspension forces with respect to 3D suspension model of VAMPIRE. Therefore, it is concluded that this dynamic model can be used both in estimation and simulation.

\section{Unscented Kalman filter and estimation results}

The detailed explanations of Unscented Kalman filter for state and parameter estimation with some examples are reported by several authors [23, 25, 26, 29]. Consider the continuous time nonlinear system given in Equation 5 in the form as stated by Zheng et al. [44]:

$$
\begin{aligned}
& \dot{x}(t)=f(x(t), u(t), \theta)+q(t), \\
& y(t)=h(x(t), \theta)+r(t),
\end{aligned}
$$

where $x(t) \in \mathbb{R}^{n}$ is the system state vector, $u(t) \in \mathbb{R}$ is the input (in this case lateral alignment irregularities), $\theta$ is the parameter (i.e. maximum friction coefficient), $y(t) \in$ $\mathbb{R}^{m}$ is the measurement vector (i.e. system output), $q(t) \sim N(0, Q(t))$ and $r(t) \sim$ $N(0, R(t))$ are process and measurement noises, respectively. In this study, the system is considered as discretized continous system and can be expressed by

$$
\begin{aligned}
& x_{k}=f_{d}\left(x_{k-1}, u_{k-1}, \theta_{k-1}, k-1\right)+q_{k-1}, \\
& y_{k}=h\left(x_{k}, \theta_{k-1}, k-1\right)+r_{k},
\end{aligned}
$$

where $x_{k}=x(k T)$, the subscript $k$ represents discrete time, and $T$ is the sampling period. The function $f_{d}$ does not require an explicit formula, but a numerical integration is needed for calculation. In this study, fourth order Runge-Kutta numerical integration method is used. Additionally, the sampling rate is $1 \mathrm{kHz}$, which is a valid sampling rate in dynamic analysis of railway vehicles as stated in chapter 2 of the study presented by Garg and Dukkipati [41]. In this case of application, the input to the system is lateral track irregularities, but as the measurements are taken from wheelset, it is not included in the system. Therefore, the system is output only. A 
non-augmented (i.e. additive noise case) joint unscented Kalman filter structure is used since it has an advantage of less computational complexity. The state vector is chosen similar to previous studies reported by Hubbard et al. [10, 22], whereas in this study contact force and moment are not included in the state vector since direct estimation of friction condition is aimed. State vector can be given as

$$
\hat{x}=\left[\begin{array}{lllll}
\hat{y} & \hat{\psi} & \dot{\hat{y}} & \dot{\hat{\psi}} & \hat{\mu}_{0}
\end{array}\right] .
$$

It is emphasized by Ward et al. [9] and Hubbard et al. [10, 22] that all states of wheelset dynamics are essential for estimation. Therefore, wheelset positions and velocities are required as measurements. Especially, these data could be derived from accelerometers and yaw gyros [10], and such a method is given for estimating rail irregularities by Kawasaki and Youcef-Toumi [45]. This method can also be used for deriving positions and velocities of a wheelset. Therefore, derivation of positions and velocities from accelerometers and yaw gyros is not investigated in this study. In addition to state vector, output vector (i.e. measurements vector) can be given as

$$
y_{\text {out }}=\left[\begin{array}{llll}
y & \psi & \dot{y} & \dot{\psi}
\end{array}\right] .
$$

Readers are highly referred to read the study by Matzuka et al. [29], which demonstrates the use of Kalman type filters for parameter estimation including UKF.

A series of simulations are carried out by using unscented Kalman filter. Firstly, static tests are given. The word static refers to the friction conditions which no friction change in the interface occur. The friction conditions, which are demonstrated in Table 1 , are used in simulations. For static tests, initial conditions for states are same and equal to zero. Initial parameter estimates $\hat{\mu}_{0}=\left[\begin{array}{llll}0.10 & 0.15 & 0.15 & 0.40\end{array}\right]$ are selected with respect to the real friction conditions $\mu_{0}=\left[\begin{array}{llll}0.04 & 0.08 & 0.20 & 0.50\end{array}\right]$. Furthermore, the filter parameters $\alpha$ which is used to determine distribution of sigma points, $\kappa$ the secondary scaling parameter, and $\beta$ a non-negative weight incorporating prior knowledge of state distribution are taken $0.5,0$ and 2, respectively. Initial covariance matrix, process and measurement noise matrices considered here are the same for all conditions and given by

$$
\begin{aligned}
P_{0} & =\left[\begin{array}{lll}
10^{-3}, & 10^{-3}, 10^{-3}, 10^{-3}, \quad 0.5
\end{array}\right], \\
Q & =\left[10^{-3}, 10^{-3}, 8 \times 10^{-4}, 8 \times 10^{-4}, 1.6 \times 10^{-1}\right], \\
R & =\left[10^{-8}, 10^{-8}, 10^{-8}, 10^{-8}\right] .
\end{aligned}
$$

During simulations, it has been observed that for dry and low friction conditions, parameter term of the state vector can be unrealistic. This is due to some sigma points either include very high level of maximum friction coefficient estimates at dry conditions or very low level of maximum friction coefficient estimates (even negative values) at low and very low friction conditions. In order to prevent this unrealistic situation, the box constraint mentioned by Kandepu et al. [28] is applied to parameter estimate. Parameter estimate is controlled with respect to the box constraint which can be presented as 


$$
0.01 \leq \hat{\mu}_{0} \leq 0.60
$$

These limits for maximum friction coefficient, which are applied as a box constraint, are the realistic limits that corresponds to the real situation in wheel-rail interface. Results for static tests can be seen in Figure 6. From this figure, it can be rapidly concluded that the estimator provides good estimation of the friction levels. There is no significant differences between estimates and real values. However, estimator has a difficulty to converge exact values of the parameter due to the similar dynamic responses for close friction coefficients in magnitude (e.g. for 0.45 and 0.5 ). Such situation is also reported in Figure 10 of previous study by Hubbard et al. [22]. Furthermore, in order to interpret outputs of static tests clearly, a moving average window [9] can be used to eliminate drifts in the parameter estimate.

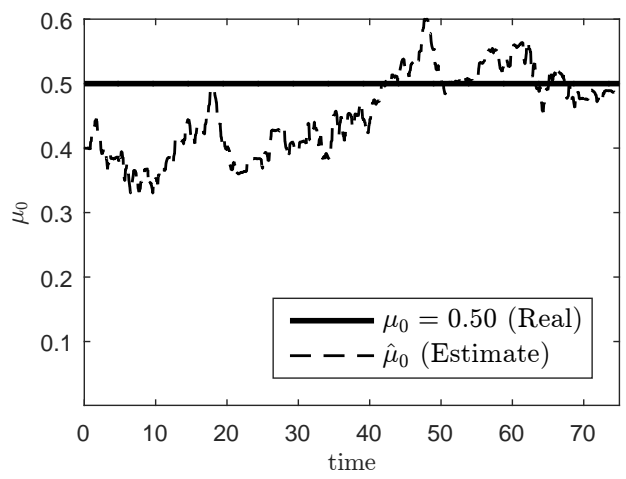

(a)

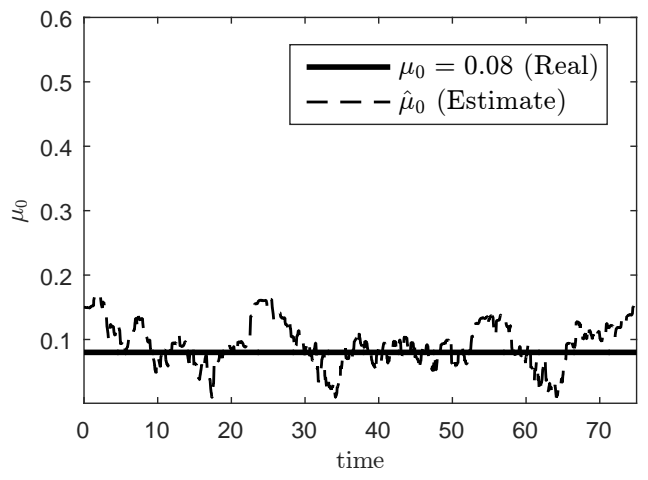

(c)

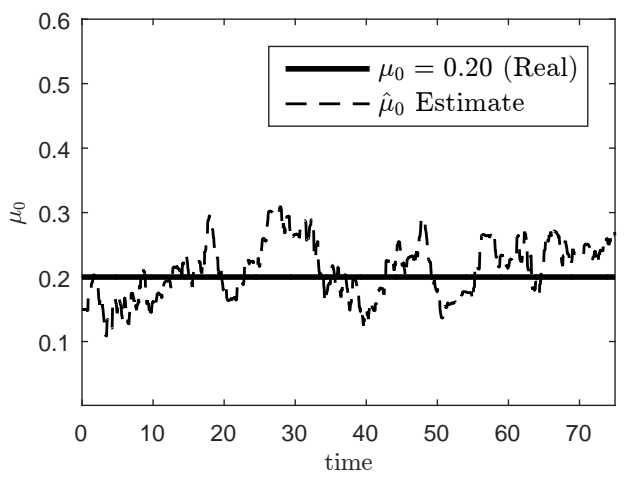

(b)

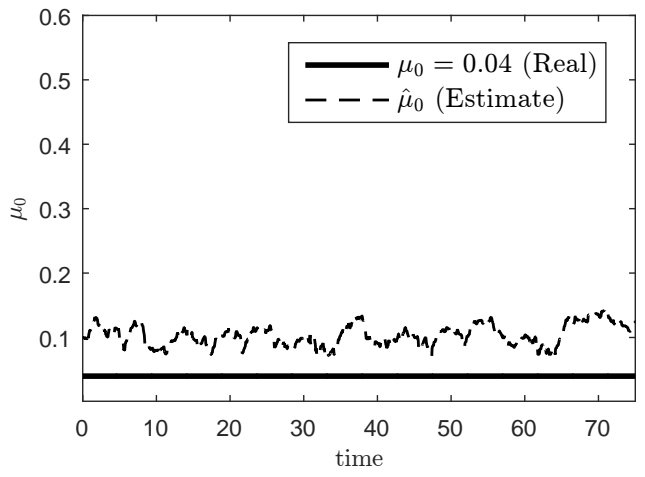

(d)

Figure 6.: Maximum friction coefficient estimation results in static case

Last remark about the static tests is, for very low friction condition (i.e. $\mu=0.04$ ), wheelset is operated close to the instability due to considered reduction factors $k_{A}$ and $k_{S}$ and combined with the structure of the joint unscented Kalman filter, this situation causes performance degradation in the estimation of very low friction condition. Due to the structure of joint unscented Kalman filter, the error is equally partitioned among the states and the parameter, as expressed by Matzuka et al. [29]. The effect of these facts can be observed in Figure 6 (d). However, the performance of the estimator is sufficient to detect a friction level which is under the critical friction level for braking $(\mu=0.15)$ mentioned in the last paragraph of Section 2 . 
For such estimators, a visual inspection of the results can be deceptive. Therefore, a performance measure different from visual inspection should be considered. The performance measures for family of Kalman filters are provided by Haug [30]. In this study, root mean squared error is used as the performance measure. Root mean squared error can be given as

$$
e_{R M S}=\left[\frac{1}{M} \sum_{i=0}^{M}\left(\theta_{i}-\hat{\theta}_{i}\right)^{2}\right]^{\frac{1}{2}}
$$

where $\theta$ represents the parameter (i.e. $\left.\mu_{0}\right)$ and $M$ represents the total number of sampled points. For the first case given in Fig. 6 (d) (i.e. $\mu_{0}=0.04$ ), for 75 sec. simulation, the Root Mean Squared Error (i.e. RMSE) is approximately 0.0654. The level of RMSE in this case is satisfactory since the detection of very low conditions is sufficient with respect to the critical friction coefficient value $(\mu=0.15)$ for braking. When quantities of the friction levels are considered, the RMSE levels show that this one step estimation scheme is promising. RMSEs for different friction levels can be found in Table 4.

Table 4.: RMSE values for static tests

\begin{tabular}{ccccc}
\hline & Dry & Wet & Low & Very low \\
\hline Root mean squared errors & 0.0781 & 0.0457 & 0.0367 & 0.0654 \\
\hline
\end{tabular}

As well as adaptation to static friction conditions, estimator should be able to adapt itself to a change in friction conditions. Therefore, a series of step change tests are conducted and results can be seen in Figure 7. All initial conditions and covariance matrices for the step change in friction tests are same as they are in static tests shown in Figure 6. The difference in step change tests is that at $t=20$ seconds the maximum friction coefficient changes to low (i.e. $\mu_{0}=0.08$ ) and very low (i.e. $\left.\mu_{0}=0.04\right)$ friction conditions. Additionally, for the first 20 seconds, the results for dry and wet conditions in step change tests are same as the case in static tests. It is revealed in Figure 7 that estimator can adapt the maximum friction coefficient estimate from dry and wet conditions to low and very low friction conditions. Most important point in this approach is that the estimator is robust against the change in other parameters of the creep force and friction model. With decreasing friction conditions, ratio of friction coefficients $\left(\mu_{0} / \mu_{\infty}\right) A$, coefficient of exponential friction decrease $B$, reduction factors $k_{A}$ and $k_{S}$ decrease as well, but estimator starts with the knowledge of these parameters for dry and wet conditions. In other words, after 20 seconds these parameters also change, whereas no information about these changes is provided to estimator. Even in such condition, estimator is robust enough to handle uncertainties in other creep force and friction model parameters.

One may criticize the slow response of the estimator in case of change from dry to low and very low conditions. This is due to structure of the joint unscented Kalman filter. As stated by Matzuka et al. [29], the joint method is partitioning the error equally among the states and the parameter, and can pass it back and forth. This situation causes estimator to operate with lag and drifts in parameter estimation. A dual filter can operate without lag and drifts, but this method requires interpretation of states by using a second filter, which means post processing of states and it is not desirable. However, if the critical value of friction coefficient $(\mu=0.15)$ for braking is 


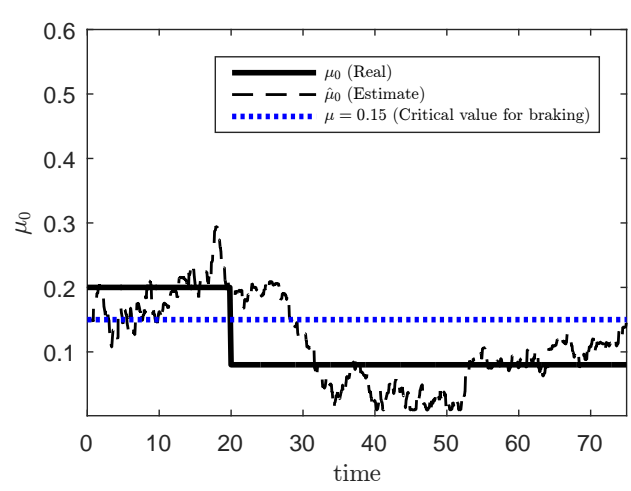

(a) From $\mu_{0}=0.20$ to $\mu_{0}=0.08$

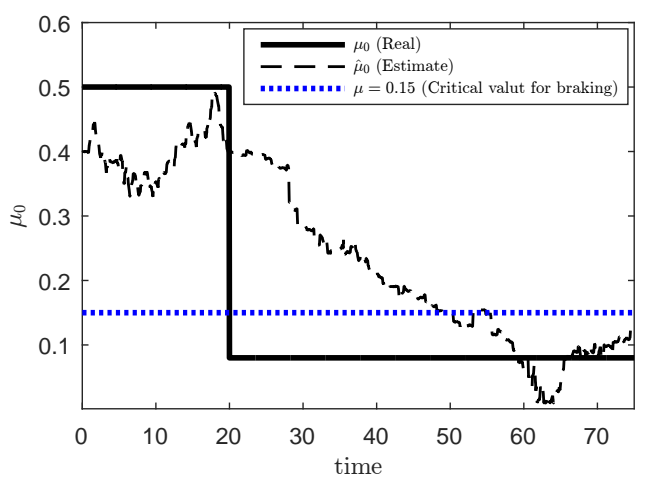

(c) From $\mu_{0}=0.50$ to $\mu_{0}=0.08$

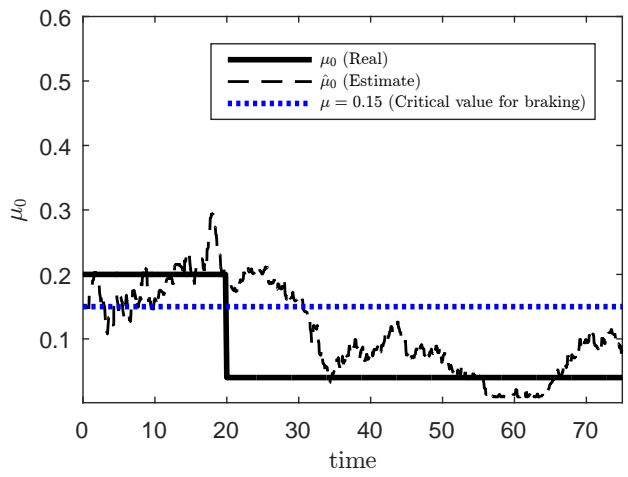

(b) From $\mu_{0}=0.20$ to $\mu_{0}=0.04$

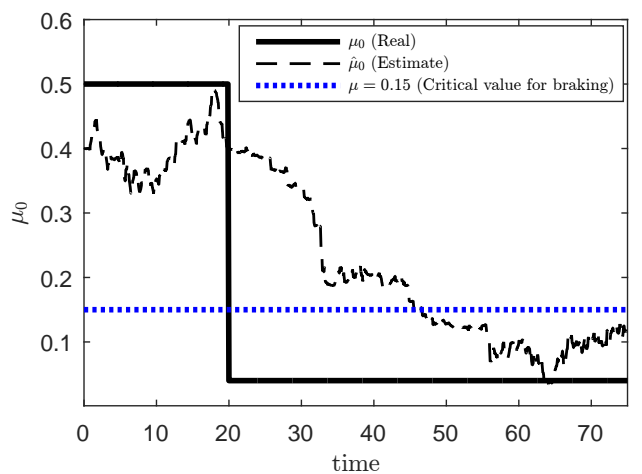

(d) From $\mu_{0}=0.50$ to $\mu_{0}=0.04$

Figure 7.: Step change in friction conditions

taken as an indicator, the performance of the estimator is considered as sufficient. In case of change from dry to low and very low conditions, estimator reaches the critical value of friction coefficient approximately 25 seconds later after step change. When the translational velocity of the vehicle $(40 \mathrm{~m} / \mathrm{s})$ is taken into account, the distance for having information about critical level is equal to 1000 meters. It is a well-known fact that in case of high speed trains, braking distances can be up to 3000 meters and even more. Therefore, the response is sufficient enough to alert driver and/or decision makers to take necessary actions within the braking distance of high speed trains in operation.

\section{Conclusion}

Low friction condition in railway vehicle systems is a problem as they can cause problems in accelerating and braking which are the possible reasons of wear, unstability of vehicle and unpredictable delays in schedule. Furthermore, important track signals can be passed due to low friction levels and this can cause accidents. The identification of such conditions is therefore important. This study proposes the use of joint unscented Kalman filter for identification of such conditions. The novelty of using a joint unscented Kalman filter is that it eliminates the post-processing methods proposed in previous studies. The simulations presented here show that this estimation scheme is promising for estimating maximum friction coefficient. Besides, the use of real track irregularity data is an indication that from real vehicle measurements, such 
a mechanism can be used to estimate friction conditions.

It is obvious from the Figure 7 that there is a delay to alert driver. This is due to the structure of the UKF and UKF can only achieve good performance under accurate reference models, exact knowledge of noise distribution and proper initial conditions as stated by Han et al. [46]. Aside from using a dual filter for improving the detection of friction coefficient change, which means post-processing of states via second filter, adaptive mechanisms can be put into UKF to reduce the time for detection [46]. These adaptive mechanisms provide promising results and automatically tunes the filter parameters to match real statistics and conditions [46].

Traction and braking processes cause a change in creepage. This situation may cause the wheelset operate in the nonlinear region of the creep force-creepage curve and a degradation in the performance of the estimation scheme. Furthermore, especially during traction and braking processes, the wheelset can have almost same lateral dynamic responses for different friction coefficients with different tractive and braking efforts [8]. Estimator can fail to detect friction conditions accurately in such cases. Therefore, with the knowledge of tractive or braking effort and a simple torsional model as used by Hussain et al. [8], friction conditions can be detected during traction or braking by using the estimation scheme presented in this study.

Further work can be the use of adaptive mechanisms for tuning filter parameters and validation of such estimation mechanism with the data taken from a multi-body system simulation model or real vehicle measurements.

\section{References}

[1] Ward CP, Weston P, Stewart E, et al. Condition monitoring opportunities using vehiclebased sensors. Proceedings of the Institution of Mechanical Engineers, Part F: Journal of Rail and Rapid Transit. 2011;225(2):202-218.

[2] Zhongshun Z, Bowen X, Lei M, et al. Parameter estimation of a railway vehicle running bogie using extended kalman filter. In: Control Conference (CCC), 2014 33rd Chinese; IEEE; 2014. p. 3393-3398.

[3] Onat A, Voltr P, Lata M. Estimation of primary suspension parameters from lateral dynamic response of a wheelset. In: 22nd International Conference Engineering Mechanics; 2016. p. 433-436.

[4] Charles G, Goodall R. Low adhesion estimation. In: Railway Condition Monitoring, 2006. The Institution of Engineering and Technology International Conference on; IET; 2006. p. 96-101.

[5] Charles G, Goodall R, Dixon R. Model-based condition monitoring at the wheel-rail interface. Vehicle System Dynamics. 2008;46(S1):415-430.

[6] Xia F, Cole C, Wolfs P. An inverse railway wagon model and its applications. Vehicle system dynamics. 2007;45(6):583-605.

[7] Xia F, Cole C, Wolfs P. Grey box-based inverse wagon model to predict wheel-rail contact forces from measured wagon body responses. Vehicle System Dynamics. 2008;46(S1):469 479 .

[8] Hussain I, Mei T, Ritchings R. Estimation of wheel-rail contact conditions and adhesion using the multiple model approach. Vehicle System Dynamics. 2013;51(1):32-53.

[9] Ward CP, Goodall RM, Dixon R, et al. Adhesion estimation at the wheel-rail interface using advanced model-based filtering. Vehicle System Dynamics. 2012;50(12):1797-1816.

[10] Hubbard P, Ward C, Goodall R, et al. Real time detection of low adhesion in the wheel/rail contact. Proceedings of the Institution of Mechanical Engineers, Part F: Journal of Rail and Rapid Transit. 2013;:0954409713503634.

[11] Spiryagin M, Cole C, Sun YQ. Adhesion estimation and its implementation for traction 
control of locomotives. International Journal of Rail Transportation. 2014;2(3):187-204.

[12] Meymand SZ, Keylin A, Ahmadian M. A survey of wheel-rail contact models for rail vehicles. Vehicle System Dynamics. 2016;54(3):386-428.

[13] Kalker JJ. On the rolling contact of two elastic bodies in the presence of dry friction [dissertation]. TU Delft, Delft University of Technology; 1967.

[14] Kalker JJ. Three-dimensional elastic bodies in rolling contact. Springer Science \& Business Media; 1990.

[15] Hertz H. Über die berührung fester elastischer körper. Journal für die reine und angewandte Mathematik. 1882;

[16] Kalker J. A fast algorithm for the simplified theory of rolling contact. Vehicle system dynamics. 1982;11(1):1-13.

[17] Bosso N, Gugliotta A, Zampieri N. Rtcontact: an efficient wheel-rail contact algorithm for real-time dynamic simulations. In: 2012 Joint Rail Conference; American Society of Mechanical Engineers; 2012. p. 195-204.

[18] Bosso N, Spiryagin M, Gugliotta A, et al. Mechatronic modeling of real-time wheel-rail contact. Springer; 2013.

[19] Zhou Y, Mei T, Freear S. Real-time modeling of wheel-rail contact laws with system-onchip. IEEE Transactions on Parallel and Distributed Systems. 2010;21(5):672-684.

[20] Polach O. A fast wheel-rail forces calculation computer code. Vehicle System Dynamics. 2000;33:728-739.

[21] Polach O. Creep forces in simulations of traction vehicles running on adhesion limit. Wear. 2005;258(7):992-1000.

[22] Hubbard P, Ward C, Dixon R, et al. Models for estimation of creep forces in the wheel/rail contact under varying adhesion levels. Vehicle System Dynamics. 2014;52(sup1):370-386.

[23] Julier SJ, Uhlmann JK, Durrant-Whyte HF. A new approach for filtering nonlinear systems. In: American Control Conference, Proceedings of the 1995; Vol. 3; IEEE; 1995. p. 1628-1632.

[24] Kalman RE. A new approach to linear filtering and prediction problems. Journal of Fluids Engineering. 1960;82(1):35-45.

[25] Wan E, Van Der Merwe R. Chapter 7: The unscented kalman filter. Kalman Filtering and Neural Networks. 2001;:221-280.

[26] Van Der Merwe R. Sigma-point kalman filters for probabilistic inference in dynamic statespace models [dissertation]. Oregon Health \& Science University; 2004.

[27] Julier SJ, Uhlmann JK. Unscented filtering and nonlinear estimation. Proceedings of the IEEE. 2004;92(3):401-422.

[28] Kandepu R, Foss B, Imsland L. Applying the unscented kalman filter for nonlinear state estimation. Journal of Process Control. 2008;18(7):753-768.

[29] Matzuka B, Aoi M, Attarian A, et al. Nonlinear filtering methodologies for parameter estimation. Department of Mathematics, North Carolina State University. 2012;1.

[30] Haug AJ. Bayesian estimation and tracking: A practical guide. John Wiley \& Sons; 2012.

[31] Onat A, Voltr P, Lata M. Nonlinear wheel/rail contact geometry characteristics \& determination of hertzian contact. Scientific papers of the University of Pardubice (Series B). 2014;19:145-152.

[32] Li Z. Wheel-rail rolling contact and its application to wear simulation. TU Delft, Delft University of Technology; 2002.

[33] Arnold M, Netter H. Wear profiles and the dynamical simulation of wheel-rail systems. In: Progress in industrial mathematics at ecmi 96. Springer; 1997. p. 77-84.

[34] Schupp G, Weidemann C, Mauer L. Modelling the contact between wheel and rail within multibody system simulation. Vehicle System Dynamics. 2004;41(5):349-364.

[35] Shabana AA, Zaazaa KE, Sugiyama H. Railroad vehicle dynamics: a computational approach. CRC press; 2007.

[36] Gensys. Users Manual for Program KPF [http://www.gensys.se/doc_html/kpf.html\#jOutput]. 2016. [Online; accessed 2016-02-15].

[37] Burgelman N, Li Z, Dollevoet R. A new rolling contact method applied to conformal 
contact and the train-turnout interaction. Wear. 2014;321:94-105.

[38] Iwnicki S. Handbook of railway vehicle dynamics. CRC press; 2006.

[39] Onat A, Voltr P, Lata M. Analysis of lateral dynamical response of a wheelset during traction. In: 22nd International Conference "Current Problems in Rail Vehicles" - PRORAIL 2015; Vol. 2; 2015. p. 73-82.

[40] EN. 1302/2014/EU. Locomotives and passenger rolling stock - LOC \& PAS TSI. 2014;1.

[41] Garg VK, Dukkipati RV. Dynamics of railway vehicle systems . 1984.

[42] Karis T. Track irregularities for high-speed trains. Master of Science Thesis, Stockholm, Sweden. 2009;

[43] Pombo J, Ambrósio J, Silva M. A new wheel-rail contact model for railway dynamics. Vehicle System Dynamics. 2007;45(2):165-189.

[44] Zheng M, Ikeda K, Shimomura T. Estimation of continuous-time nonlinear systems by using the unscented kalman filter. In: SICE Annual Conference 2010, Proceedings of; IEEE; 2010. p. 2192-2195.

[45] Kawasaki J, Youcef-Toumi K. Estimation of rail irregularities. In: American Control Conference, 2002. Proceedings of the 2002; Vol. 5; IEEE; 2002. p. 3650-3660.

[46] Han J, Song Q, He Y. Adaptive unscented kalman filter and its applications in nonlinear control. INTECH Open Access Publisher; 2009. In Kalman Filter: Recent Advances and Applications. 